\title{
Rate of reinforcement and session duration as determinants of within-session patterns of responding
}

\author{
FRANCES K. MCSWEENEY \\ Washington State University, Pullman, Washington
}

\begin{abstract}
Rats pressed levers for Noyes pellets or keys for sweetened condensed milk reinforcers delivered by multiple schedules. Session length and baseline rates of reinforcement were varied in two experiments. Rates of responding increased during the early part of the session and then decreased for both responses and reinforcers, as well as for all subjects and values of the independent variables. Changes in response rates across the session sometimes exceeded $500 \%$. Response rates peaked approximately $20 \mathrm{~min}$ after the beginning of the session, regardless of session duration, when subjects responded on a multiple variable interval 1-min variable interval 1-min schedule. The function was flatter for longer sessions than it was for shorter sessions. The function was flatter, more symmetrical, and peaked later for lower rates of reinforcement than for higher rates of reinforcement. The function appeared early in training, and further experience moved and reduced its peak. Variables related to reinforcement exerted more control over some aspects of this function than did variables related to responding. These within-session patterns of responding may have fundamental implications for experimental design and theorizing.
\end{abstract}

Absolute rate of responding is one of the primary dependent variables in operant psychology. Given its importance, it is surprising that little is known about the way in which response rates change across experimental sessions.

Preliminary information on this topic was provided by McSweeney, Hatfield, and Allen (1990). In their study, rats pressed levers for Noyes pellets or pressed keys for sweetened condensed milk delivered by a multiple variable interval 1-min variable interval 1-min (multiple VI 1-min VI 1-min) schedule. The components of the multiple schedule alternated every $5 \mathrm{~min}$. Rates of responding increased for the first 20 min of the 60 -min experimental session and then decreased. This bitonic function occurred for both responses and both reinforcers, as well as for each subject. The change in response rate was large. For example, the rate of keypressing averaged 15.7 and 27.5 responses per minute for the first and last $5 \mathrm{~min}$ of the session, respectively. It averaged 70.0 responses per minute at its peak.

The bitonic functions reported by McSweeney et al. have many implications. First, they imply that the rate of responding averaged across the session-one of the primary dependent variables in operant psychologymasks large and reliable regularities in animal behavior, at least in some cases. Therefore, this molar measure must

This research was partially supported by NIMH Grant MH 42466. The author wishes to thank John Hinson for his comments on an early version of this manuscript and Cam Melville for his help in conducting the experiments. Reprints may be obtained from F. K. McSweeney, Department of Psychology, Washington State University, Pullman, WA 99164-4820. be supplemented by more molecular measures for a complete theory of behavior to emerge.

Second, the bitonic function may help to clarify some theoretical issues. For example, some theories predict that response rates will increase monotonically with increases in their rates of reinforcement (e.g., Herrnstein, 1970). Other theories predict that response rates should increase with increases in reinforcement up to a point and then decrease with further increases (e.g., Baum, 1981; Staddon, 1979). The fact that responding changes within sessions implies that there may be more than one answer to this question. For example, response rates might increase monotonically with increases in the rates of reinforcement during some parts of the session but not during others.

Third, the within-session patterns of responding reported by McSweeney et al. (1990) have methodological implications. Experiments are often conducted so that session length covaries with the independent variable. For example, when studying the effect of rate of reinforcement on rate of responding, the experimenter must allow either session length or number of reinforcers delivered per session to covary with rate of reinforcement. Session length is often varied (e.g., Catania \& Reynolds, 1968), because varying the number of reinforcers might produce systematic changes in satiation. However, allowing session length to covary with the independent variable may also yield uninterpretable average rates of responding if response rates change systematically across the session. If, for example, response rates declined across the session, then shorter sessions might yield higher average rates of responding than might longer sessions. The effect of the independent variable on the average rate of respond- 
ing would not be interpretable if sessions of different length were conducted for different values of the independent variables.

Finally, within-session patterns of responding have implications for the use of within-session procedures. Withinsession procedures present different aspects of an independent variable in different parts of a single experimental session rather than in different sessions (e.g., Heyman, 1983; McSweeney \& Melville, 1990; Staddon, 1967). Such procedures are useful because the large shifts in the baseline response rates that can occur across sessions do not add variability to their data (e.g., McSweeney, Dougan, Higa, \& Farmer, 1986; Spealman \& Gollub, 1974). Such procedures also save time. All values of the independent variable are conducted at once, rather than successively. Saving time is always convenient. It may also be necessary when examining the behavior of a species of animal with a short lifespan such as the rat. However, the effect of the independent variable will also be difficult to interpret if the within-session procedure presents different values of its independent variable at times within the session that control different response rates.

The factors that control within-session patterns of responding deserve study because of the large size of the effect, its reliability across subjects, responses and reinforcers, and its potential theoretical and methodological implications. The present experiments examine two factors that might control these patterns: rate of reinforcement and session length. Experiment 1 repeats McSweeney et al.'s (1990) experiment on leverpressing but varies the baseline rate of reinforcement. Experiment 2 repeats their experiment on keypressing but varies session duration.

\section{EXPERIMENT 1}

Experiment 1 examined within-session patterns of responding as a function of rate of reinforcement when rats press levers for Noyes pellets.

\section{Method}

Subjects. The subjects were 5 experimentally naive rats, from Sprague-Dawley stock, bred in the Johnson Tower Vivarium at Washington State University. They were approximately 120 days old at the beginning of the experiment and were maintained at approximately $80 \%$ of their free-feeding body weights.

Apparatus. The apparatus was a standard two-lever experimental enclosure for rats $(20 \times 24.5 \times 24.5 \mathrm{~cm})$. A $5 \times 5.5 \mathrm{~cm}$ opening, which allowed access to food reinforcers (45-mg Noyes pellets), was centered on the front panel, $0.5 \mathrm{~cm}$ above the floor. Two $4 \times 1.5 \mathrm{~cm}$ levers were located $2.5 \mathrm{~cm}$ from this opening, one on each side. The levers were $5 \mathrm{~cm}$ above the floor and extended $1.5 \mathrm{~cm}$ into the enclosure. A light ( $2 \mathrm{~cm}$ in diameter) was located $2.5 \mathrm{~cm}$ above each lever. A third light $(2 \mathrm{~cm}$ in diameter) was centered in the front panel, $4 \mathrm{~cm}$ from the ceiling. The houselight $(2 \mathrm{~cm}$ in diameter) was located in the center of the ceiling.

The apparatus was enclosed in a sound-attenuating chamber. A ventilating fan masked noises from outside of the apparatus. A SYM microcomputer, located in another room, presented the experimental events and recorded data.

Procedure. The subjects were shaped by successive approximations to press the right lever. The rate of reinforcement was gradu- ally decreased until the subjects responded on a VI 30-sec schedule. They were then placed on the experimental procedure.

The experimental procedure presented a multiple schedule in which the components alternated every $5 \mathrm{~min}$. The lights above the right lever were illuminated during the first component, but not during the second. Reinforcers consisted of one $45-\mathrm{mg}$ Noyes pellet and were scheduled for pressing the right lever, according to a 25 interval Fleshler and Hoffman (1962) series. Sessions ended when 12 components had been presented $(60 \mathrm{~min})$. Sessions were conducted five to six times per week. The houselight was illuminated throughout the session.

The subjects began the experiment responding on a multiple VI $30-\sec$ VI $30-\mathrm{sec}$ schedule. They were then placed on a multiple VI 2-min VI 2-min, a multiple VI 4-min VI 4-min, a multiple VI 15-sec VI 15-sec, and a multiple VI 1-min VI 1-min schedule. The subjects responded on each schedule for $\mathbf{3 0}$ sessions.

\section{Results and Discussion}

Table 1 presents the mean rates of responding by each subject during each schedule. Rates are reported in responses per minute and have been calculated by dividing the total number of responses during a session by the total session time minus the time for which the magazine was available. The means are the average of the rates during the last five sessions for which each schedule was available.

Table 1 shows that responding did change with changes in the schedule. A one-way within-subject analysis of variance (ANOVA) applied to these response rates was significant $[F(4,16)=4.49]$. Here and throughout this paper, results will be considered to be significant when $p<.05$.

In general, response rates increased with increasing rates of reinforcement. The results of $t$ tests for matched pairs showed that the rate of responding during the multiple VI 4-min VI 4-min schedule was significantly lower than that during each of the other schedules except the multiple VI 60 -sec VI $60-\mathrm{sec}[t(4)=2.24$, for multiple VI 15-sec VI 15-sec; $t(4)=3.03$, for multiple VI 30-sec VI $30-\mathrm{sec} ; t(4)=4.04$, for multiple VI 2-min VI 2-min]. Responding decreased again at the highest rate of reinforcement. The rates of responding during the multiple VI 15-sec VI 15-sec schedule were significantly lower than those during the multiple VI 30-sec VI 30-sec schedule $[t(4)=-3.92]$. This decrease in responding at very high rates of reinforcement is frequently observed (e.g., Dougan \& McSweeney, 1985; McSweeney \& Melville, 1990) and is consistent with several theories (e.g., Baum, 1981; Staddon, 1979).

Table 1

Mean Rates of Responding (Responses per Minute) by Each Subject Responding on Each Schedule Over the Last Five Sessions for Which the Schedule Was Available in Experiment 1

\begin{tabular}{cccccc}
\hline & \multicolumn{5}{c}{ Schedule } \\
\cline { 2 - 5 } Subject & VI 15 & VI 30 & VI 60 & VI 120 & VI 240 \\
\hline 201 & 23.4 & 40.9 & 35.8 & 32.6 & 13.0 \\
202 & 47.3 & 66.9 & 63.9 & 38.2 & 17.9 \\
203 & 47.0 & 76.8 & 23.6 & 23.7 & 8.8 \\
204 & 25.7 & 28.8 & 24.2 & 19.2 & 18.3 \\
205 & 32.1 & 46.5 & 27.3 & 49.2 & 34.3 \\
Mean & 35.1 & 52.0 & 35.0 & 32.6 & 18.5 \\
\hline
\end{tabular}




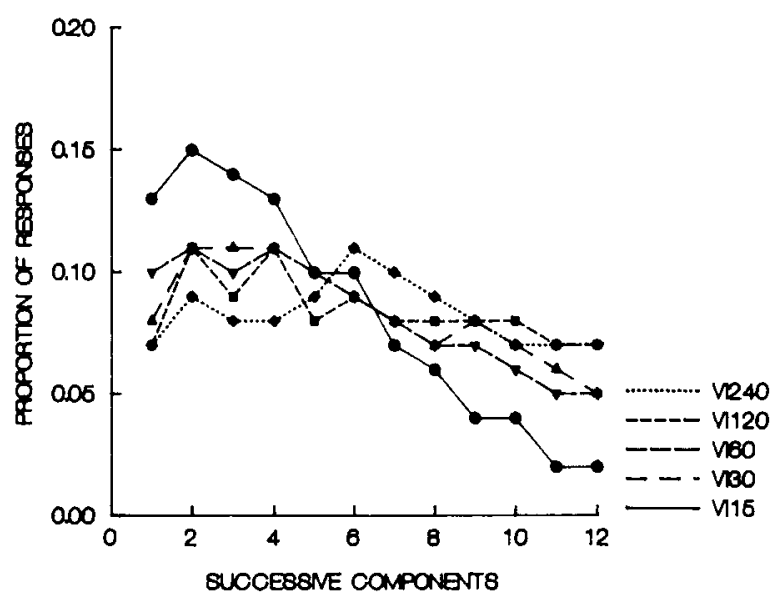

Figure 1. The proportion of total-session responses during successive components for the mean of all subjects responding in Experiment 1. Each function presents the results for a particular schedule. Results have been averaged over all subjects and over the last five sessions for which each schedule was presented.

Figure 1 presents the proportion of total-session responses during successive components for the mean of all subjects responding on each of the five schedules. Proportions have been calculated by dividing the number of responses during a component by the total number of responses during the session. The results are those for the mean of the last five sessions for which each schedule was available.

Figure 2 shows that the results presented in Figure 1 also represent those for individual subjects. It presents the proportion of the total-session responses by individual subjects during successive components within the session. Each set of axes presents the results for a particular schedule. Each function presents the results for a single subject. All proportions are the means of those recorded during the last five sessions for which each subject responded on each schedule.

Figures 1 and 2 show that the bitonic function became flatter as the programmed rate of reinforcement decreased. For example, the proportions reported in Figure 1 varied from 0.02 to 0.15 for the multiple VI $15-\mathrm{sec}$ VI $15-\mathrm{sec}$ schedule, but they varied from only 0.07 to 0.11 for the multiple VI 4-min VI 4-min schedule.

The functions also became more symmetrical around the middle of the session as the programmed rate of reinforcement decreased. Wilcoxon signed ranks tests showed that the subjects responded significantly more during the first half of the session than they did during the second half for the VI 15-sec $(p=.04)$, VI 30-sec $(p=.04)$, and VI $60-\sec (p=.04)$ schedules. The proportion of responses during the two halves did not differ significantly for the VI 2-min $(p=.07)$ or the VI 4-min $(p=.89)$ schedules.

Figures 1 and 2 also show that the peak rate of responding occurred later in the session as the programmed rate of reinforcement decreased. For the multiple VI 15 -sec
VI 15-sec schedule, the subjects' highest proportion of total responses was during the first or second component. For the multiple VI 4-min VI 4-min schedule, the subjects' highest proportion of total responses was during the sixth component.

Figure 3 presents the proportion of the total-session responses during successive components for the first recorded session of training (circles) and for the mean of the last five sesions of responding (triangles) for the multiple VI 30-sec VI 30-sec schedule. Data are plotted for the mean of all subjects. Data have been presented for the multiple VI 30-sec VI 30-sec schedule because it was the first schedule presented to the subjects. Only sessions of shaping preceded the first session with this schedule. Figure 3 shows that the bitonic function was present as early in training as the first recorded session. The effect of further training was to flatten the function and to move its peak.

The results presented in Table 1 show that the mean response rates calculated over the entire session decreased at the highest programmed rate of reinforcement. The question arises whether response rates would increase as a monotonic function of rate of reinforcement if the changes in response rates across the session were prevented.

Figure 4 answers this question. It presents the average rate of responding over the whole session (Table 1), plotted as a function of the programmed rate of reinforcement. These data appear on the top left axes and are labeled MEAN. It also presents the mean rates of responding during the $1 \mathrm{st}, 3 \mathrm{rd}$, 9th, and 12 th components, plotted as a function of the programmed rates of reinforcement. These results appear on the other set of axes. All results are those for the mean of all subjects.

Figure 4 shows that response rates increased monotonically with increases in the rate of reinforcement during the first component. Response rate declined at the highest rates of reinforcement for all other components. This suggests that the factors that produce the decrease in responding at the highest rate of reinforcement are not present at the beginning of the session. The exact nature of these variables is not known because, as will be discussed, the factors that produce the present changes in responding across the session are not known.

\section{EXPERIMENT 2}

Experiment 2 examined within-session patterns of responding as a function of session length when rats press keys for sweetened milk reinforcers.

\section{Method}

Subjects. The subjects were 5 experimentally naive rats from Sprague-Dawley stock, bred in the Johnson Tower Vivarium at Washington State University. They were approximately 120 days old at the start of the experiment and were maintained at approximately $80 \%$ of their free-feeding body weights.

Apparatus. The apparatus was a $21 \times 21.5 \times 24 \mathrm{~cm}$ enclosure, equipped with a lever and a key. An opening $(6 \mathrm{~cm}$ in diameter) allowed access to a dipper. The opening was centered in the panel, 


\section{$\vee 15-S$}

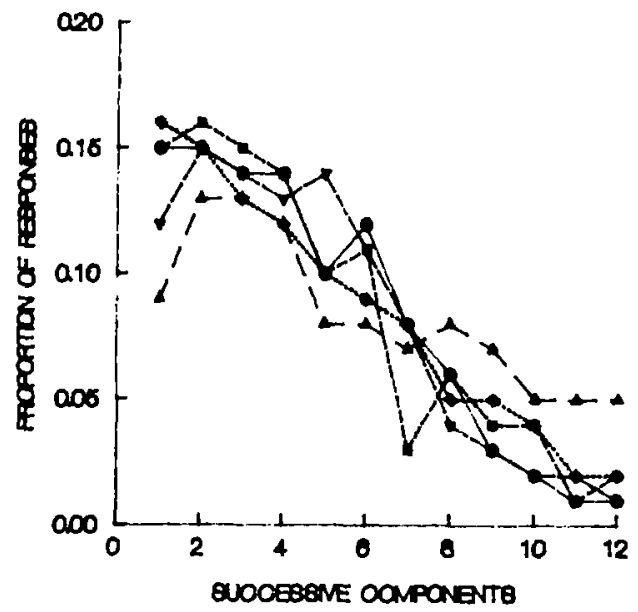

$\vee 160-S$

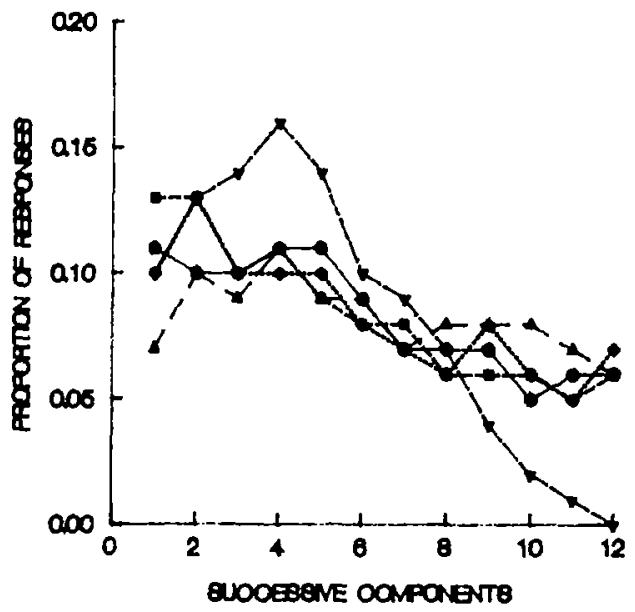

$\checkmark 130-S$

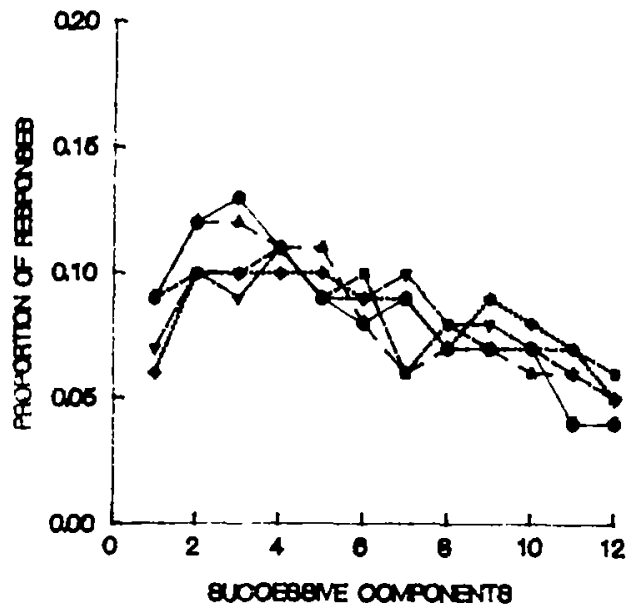

$\checkmark 120-5$

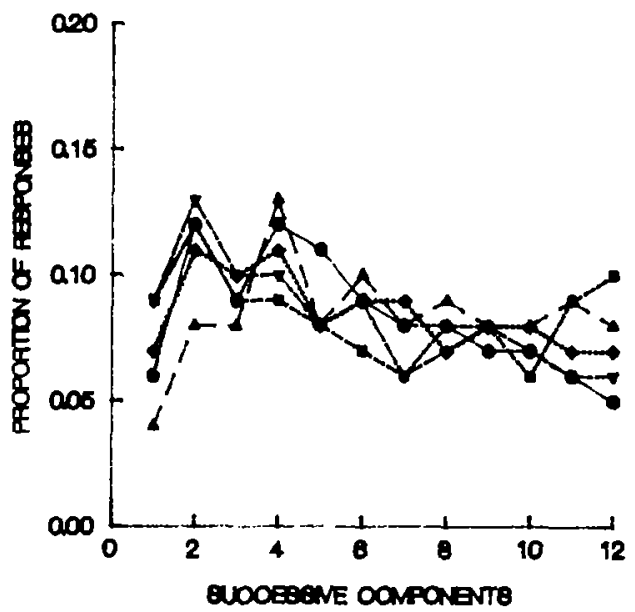

$\vee 1240-S$

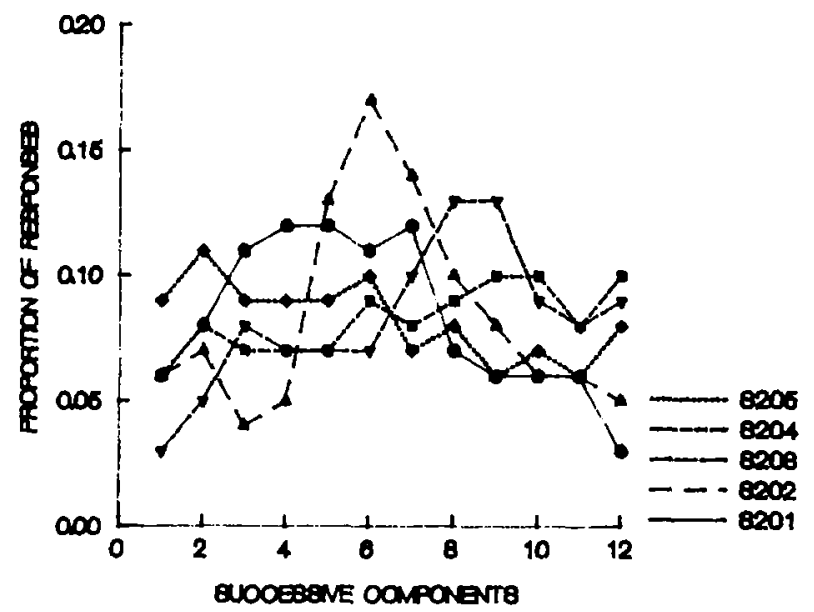

Figure 2. The proportion of total-session responses by individual subjects during successive components for each schedule conducted in Experiment 1. Each set of axes presents the results for a particular schedule. Each function presents the results for an individual subject. All proportions are the mean over the last five sessions for which each schedule was conducted. 


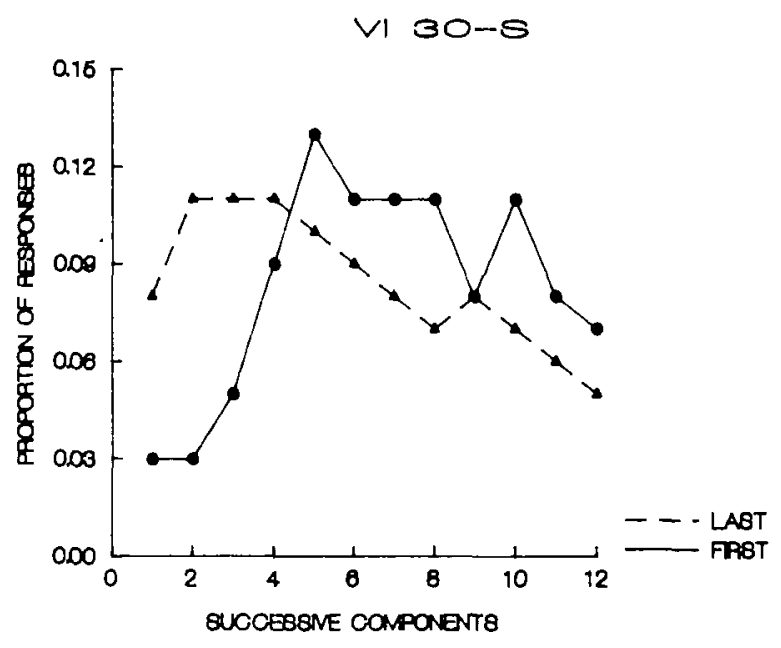

Figure 3. The proportion of total-session responses during successive components of the multiple VI 30-sec VI 30-sec schedule in Experiment 1. Results are presented for the first recorded session of training (circles) and for the mean of the last five sessions of training (triangles) for the mean of all subjects.

$4 \mathrm{~cm}$ above the floor. A $5-\mathrm{W}$ light ( $2 \mathrm{~cm}$ in diameter) was located $3 \mathrm{~cm}$ from each side of the panel and $5.5 \mathrm{~cm}$ from the top. The left light was clear, and the right light was opaque. A key $(2.5 \mathrm{~cm}$ in diameter) was located $2 \mathrm{~cm}$ below the left light. A $3.5-\mathrm{cm}$ lever, which extended $2 \mathrm{~cm}$ into the chamber, was located $3 \mathrm{~cm}$ below the right light.

The apparatus was enclosed in a sound-attenuating chamber. A ventilating fan masked noises from outside of the apparatus. A SYM microcomputer, located in another room, presented the experimental events and recorded the data.

Procedure. The procedure was identical to that of Experiment 1, with the following exceptions. The subjects pressed a key for reinforcers delivered by a multiple VI 1-min VI 1-min schedule. Reinforcers consisted of $5 \mathrm{sec}$ of access to sweetened condensed milk, mixed one to one with water. Finally, session length was varied by varying the length of the components. The following session lengths were conducted in the following order: $60 \mathrm{~min}(300 \mathrm{sec})$, $30 \mathrm{~min}(150 \mathrm{sec})$, and $90 \mathrm{~min}(450 \mathrm{sec})$. The component duration used to produce that session length appears in parentheses after the session length. The subjects responded on each session duration for 30 sessions.

\section{Results and Discussion}

Table 2 presents the mean rates of responding during the session for each of the session lengths. Response rates have been calculated as they were for Table 1 . A oneway within-subject ANOVA showed that response rates changed significantly with session length $[F(2,8)=4.87]$. The rates of responding during the 60 -min sessions were significantly greater than the rates of responding during the $30-\min [t(4)=-2.84]$ and $90-\min [t(4)=4.11]$ sessions.

Figure 5 presents the proportion of total-session responses during successive components for the mean of all subjects responding at each session length. Proportions have been calculated over all subjects and over the last five sessions for which each session length was available.
Figure 6 shows that the results presented in Figure 5 represent those for individual subjects. It presents the proportion of the total-session responses during successive components by individual subjects. Each set of axes presents the results for a particular session length. Individual functions present the results for individual subjects responding over the last five sessions for which that session length was available.

Figures 5 and 6 show that responding peaked during later components for the shorter sessions than for the longer sessions. As a result, peak response rates occurred approximately the same number of minutes after the beginning of the session, regardless of session length. According to Figure 6, responding peaked between 12.5 and 22.5 min (Components 6-9) for the 30-min sessions, between 15 and $20 \mathrm{~min}$ (Component 4) for the 60 -min sessions, and between 15 and 37.5 min (Components 3-5) for the 90 -min sessions.

The functions reported in Figures 5 and 6 were flatter for longer sessions than they were for shorter sessions. The proportion of responses during the components in Figure 5 varied from 0.02 to 0.11 for the 30 -min sessions. They varied from only 0.05 to 0.11 for the 60 - and 90 min sessions.

The functions were also more symmetrical around the middle of the session for intermediate session durations (60 $\mathrm{min}$ ) than they were for shorter and longer sessions. Wilcoxon signed ranks tests showed that the proportion of responses during the first and second halves of the sessions differed significantly for the 30-min $(p=.04)$ and 90 -min $(p=.04)$ sessions, but not for the 60-min sessions $(p=.08)$. There were more responses during the second half of the session than there were during the first half of the session for the 30 -min sessions. There were more responses during the first half than there were during the second half for the 90 -min sessions.

Figure 7 presents the proportion of the total-session responses during successive components for the first recorded session of training (circles) and for the mean of the last five sessions of responding (triangles) for the subjects responding during the $60-\mathrm{min}$ sessions. The results are those for the mean of all subjects. Data have been presented for the 60-min sessions because the subjects responded first on these sessions. They were preceded only by earlier sessions of shaping. Figure 7 shows that the bitonic function was present even during the first recorded session. The effect of further training was to flatten the function and to move its peak.

\section{GENERAL DISCUSSION}

The present results add to those of McSweeney et al. (1990) in showing that responding changes in an orderly way within sessions, at least when rats press levers or keys for food reinforcers delivered by multiple schedules. Response rates increased up to a point and then decreased. This change in response rates was large. It occurred for 


\section{MEAN}

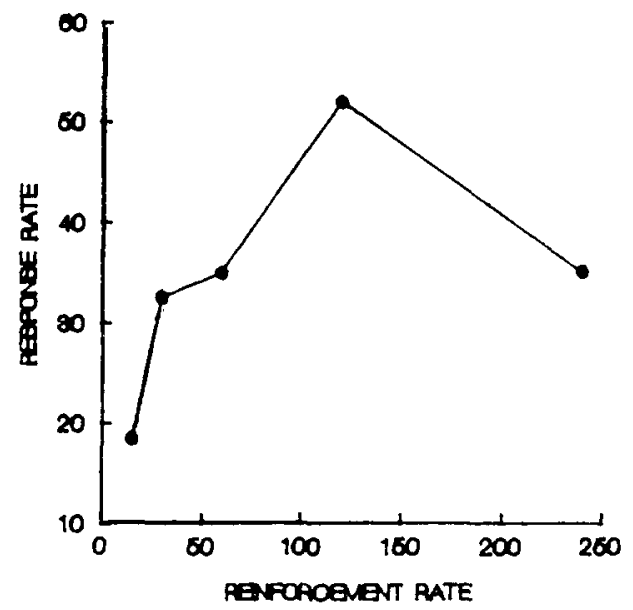

THIRD

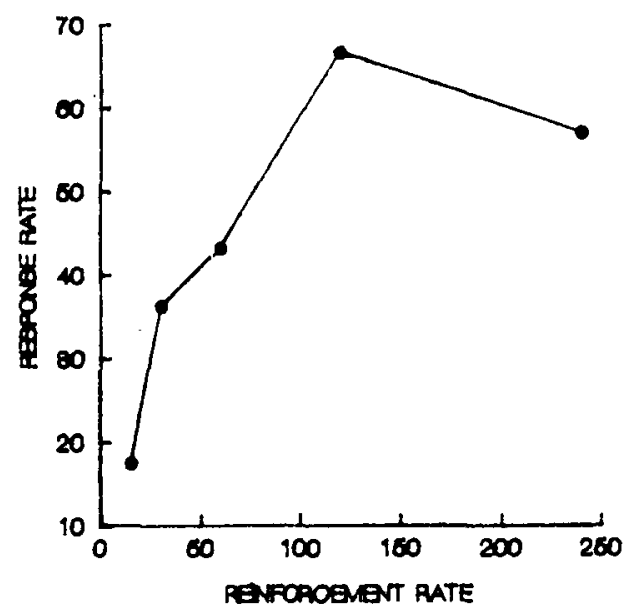

TИELFTH

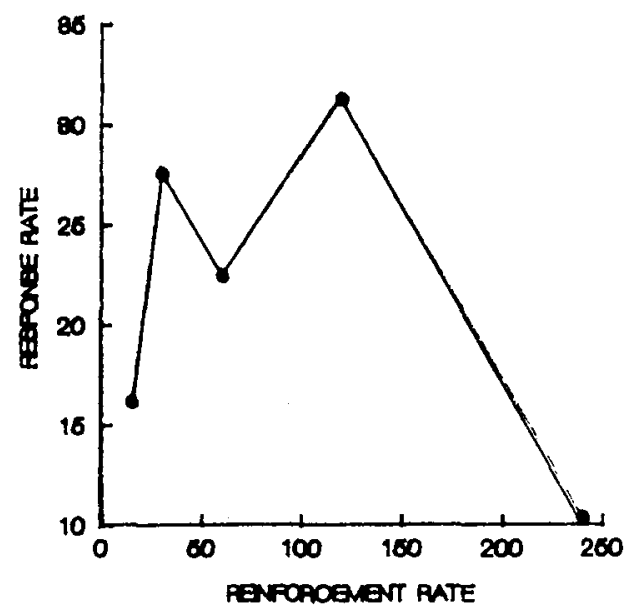

$$
\text { FIRST }
$$

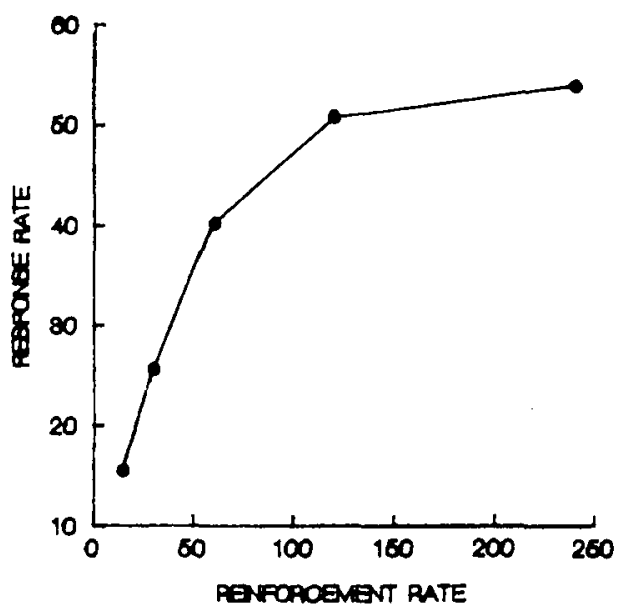

$$
\text { NINTH }
$$

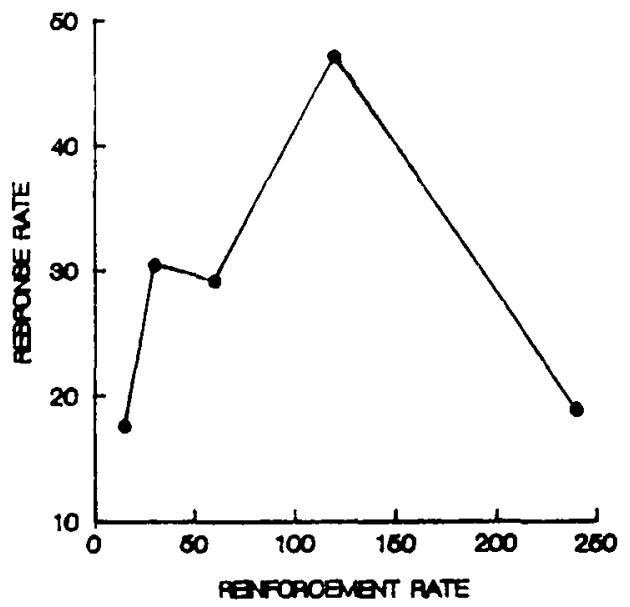

Figure 4. Rates of responding in Experiment 1, plotted as a function of programmed rates of reinforcement. The top left axes present rates of responding averaged across the entire session. The other axes present response rates for the 1st, 3rd, 9th, and 12 th components. All results are those for the mean of all subjects averaged over the last five sessions for which each schedule was available. 
Table 2

Mean Rates of Responding (Responses per Minute) by Each Subject Responding During Sessions of Each Length in Experiment 2

\begin{tabular}{cccc}
\hline & \multicolumn{3}{c}{ Session Length } \\
\cline { 2 - 4 } Subject & $30 \mathrm{~min}$ & $60 \mathrm{~min}$ & $90 \mathrm{~min}$ \\
\hline 61 & 14.0 & 80.5 & 64.4 \\
62 & 71.6 & 84.1 & 51.4 \\
63 & 31.7 & 48.7 & 37.4 \\
64 & 36.0 & 57.7 & 40.0 \\
65 & 31.3 & 53.0 & 44.5 \\
Mean & 36.9 & 64.8 & 47.5 \\
\hline
\end{tabular}

both responses and reinforcers, as well as for all subjects, rates of reinforcement, and session durations. The change in response rates appeared in steady-state behavior as well as in responding early in training.

Because of the size and reliability of this effect, the results have important implications for the behavior of rats pressing levers or keys for food reinforcers delivered by multiple schedules. The implications of these results for other literatures are not known because the generality of the bitonic function is not known. Some evidence indicates that the bitonic function is somewhat general. The results of the present study and those of McSweeney et al. (1990) indicate that it occurs for two different responses, two different reinforcers, and several different rates of reinforcement and session durations. The function has also been observed when pigeons peck keys on concurrent schedules or press treadles on positive conditioned suppression procedures (McSweeney \& Hinson, in press). On the other hand, the bitonic function might be larger for multiple schedules than for other procedures. For example, changes in the discriminative stimuli provide cues to time in the session on multiple schedules that are not present on other schedules. The discussion that follows will examine the implications of the present findings for

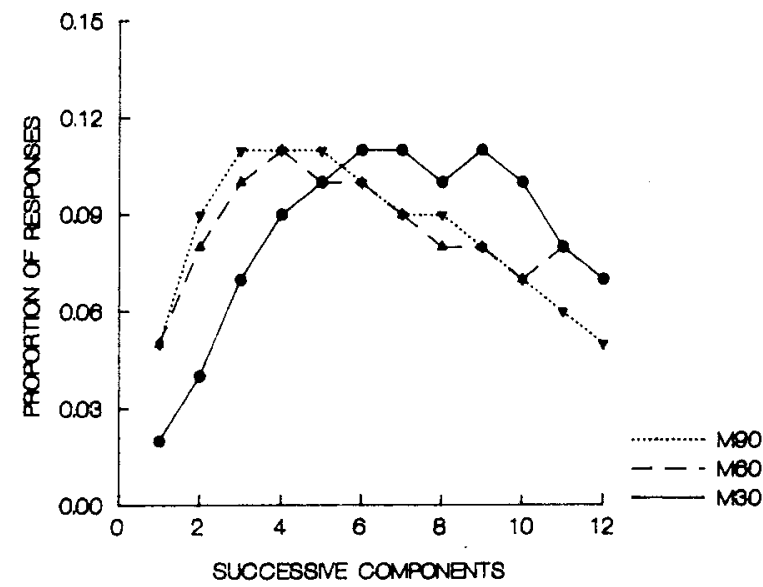

Figure 5. The proportion of total-session responses during successive components for the mean of all subjects responding in Experiment 2. Each function presents the results for a different session length. Results have been averaged over all subjects and over the last five sessions for which each session length was available. the multiple-schedule literature. Although preliminary results indicate that the function has some generality, only further experiments can determine whether these conclusions also apply to the results produced by other procedures.

The present results suggest that future studies should use caution when allowing session length to covary with their independent variable, at least when rats press keys for food reinforcers. Rates of pressing changed significantly with moderate changes in session length. For example, the overall rate of responding during the session almost doubled when the session was lengthened from $30 \mathrm{~min}$ (mean $=36.9$ responses per minute) to $60 \mathrm{~min}$ (mean $=64.8$ responses per minute). Such large changes in response rates could make the effect of the independent variable impossible to interpret.

Figure 4 shows that the pattern of responding within sessions may have confused the answer to some theoretical questions. It shows that the rates of responding at the beginning of the session increase monotonically with increases in the rate of reinforcement, confirming some theories (e.g., Herrnstein, 1970). Responding later in the session increases up to a point and then decreases, confirming other theories (e.g., Baum, 1981; Staddon, 1979). These results suggest that whatever factors produced the decline in response rates at the highest rates of reinforcement were not present at the beginning of the session. Future experiments should determine the nature of these factors. As will be discussed, none of the factors that produced the present changes in response rates across the session are known at this time. Future experiments should also determine whether within-session patterns of responding have confused the interpretation of the effect of other independent variables, such as reinforcer size or delay.

The present results imply that within-session procedures should be used with caution. Figure 1 suggests that withinsession procedures are more reasonably used for lower rates of reinforcement ( 30 reinforcers per hour or lower) than they are for higher rates when rats press levers. Figure 5 suggests that within-session procedures are more reasonably used when sessions are $60 \mathrm{~min}$ long than when they are 30 or 90 min long when rats press keys. According to Figures 1 and 5, responding is more evenly distributed throughout the session for intermediate session lengths and lower rates of reinforcement. Therefore, within-session procedures may not confuse the effect of the independent variable with within-session changes in responding if they employ intermediate session lengths and lower rates of reinforcement. However, again, because the generality of the present results is not known, the precise limitations of the within-session procedures are not known. These limitations will undoubtedly depend on many aspects of the experimental design. For example, the inclusion of timeouts during the session may change the present bitonic function in unknown ways.

The present bitonic function emerged in spite of, rather than because of, the distribution of reinforcement across the session. The present VI schedules program a flat dis- 

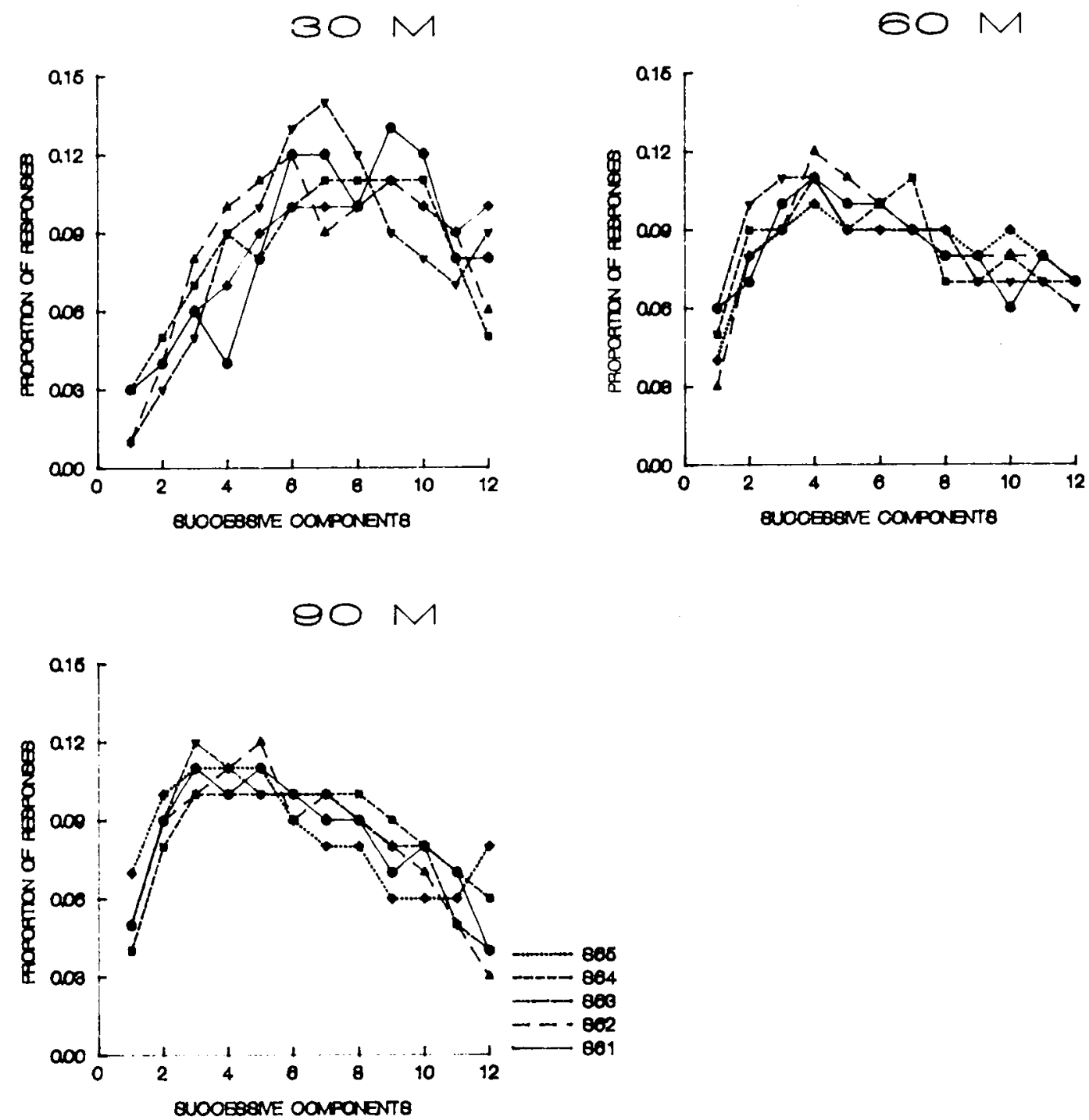

Figure 6. The proportion of total-session responses by individual subjects during successive components for each session length in Experiment 2. Each set of axes presents the results for a particular session length. Each function presents the results for an individual subject. Proportions have been averaged over the last five sessions for which each session length was available.

tribution of reinforcement across the session. Nevertheless, a bitonic function related responding to time in the session. However, the distribution of reinforcement may have influenced the bitonic function. Figures 3 and 7 showed that the bitonic function appeared early in training. The effect of further training was to flatten the function and to move its peak. The flat distribution of reinforcers aross the session may have flattened the functions. This idea could be tested by examining the effect of other distributions of reinforcers on the present functions.

The factors that produced the present functions should be determined. Two variables have traditionally described performance decrements in similar situations: fatigue and satiation. Results similar to the ascending limb of the func- tion have also been observed in two situations. First, priming has been observed when electrical brain stimulation is used as the reinforcer. Priming refers to the fact that subjects may not respond for electrical brain stimulation until some free reinforcers have been given (e.g., Olds, 1956; Olds \& Milner, 1954). Second, warm-up has been reported in many literatures. These literatures include those on the following conditioning phenomena: discriminated avoidance (e.g., Foree \& LoLordo, 1970; Hoffman, Fleshler, \& Chorny, 1961), Sidman avoidance (e.g., Powell, 1970; Wertheim, 1965), punishment (e.g., Azrin, 1960; Azrin, Holz, \& Hake, 1963; Hake \& Azrin, 1965; Hake, Azrin, \& Oxford, 1967), shock-elicited aggression (e.g., Hutchinson, Renfrew, \& Young, 1971; Lyons \& 


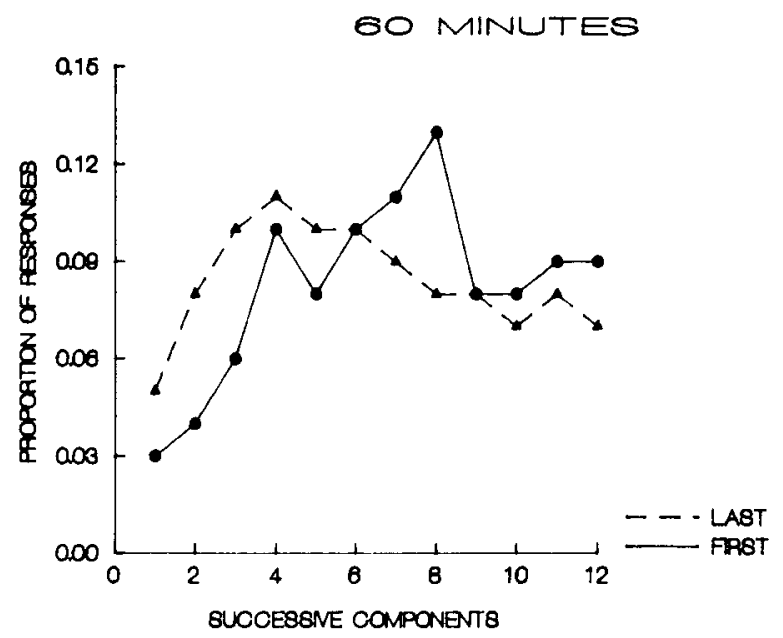

Figure 7. The proportion of total-session responses during successive components of the 60-min sessions during Experiment 2. Results are presented for the first recorded session of training (circles) and for the mean of the last five sessions of training (triangles) for the mean of all subjects.

Ozolins, 1970), and conditioned emotional response procedures (e.g., Rubin \& Brown, 1969). The early trials for such tasks as psychophysical procedures (e.g., Hodos, Leibowitz, \& Bonbright, 1976) or generalization tests (e.g., Griffin \& Stewart, 1977) may also be discarded as warm-up.

The relation between the present results and these historical observations is not known. Fatigue has not been a useful concept when describing the literature on human performance decrements (e.g., Muscio, 1921). It includes the effect of too many variables (e.g., effects at the neural, muscular, and central levels; effects resulting from overload with those resulting from underload, such as boredom). Except for warm-up for avoidance (e.g., Hineline, 1978a, 1978b), neither priming nor warm-up has been studied in systematic detail. However, what is known suggests that priming and warm-up for avoidance are not related to the present function. For example, priming is not found for all subjects. The ascending limb of the present functions is found reliably for all subjects. The duration of warm-up for avoidance is highly variable across subjects (e.g., Badia, Culbertson, \& Lewis, 1971). The ascending limb of the present functions ended at approximately the same time for all subjects. Warm-up for avoidance may also fail to appear in measures of response rate. Warm-up for avoidance refers to the fact that subjects may receive a large proportion of their total session shocks in the first few minutes of the session (e.g., Powell, 1970). Interestingly, large changes in the number of shocks have been reported in the absence of any change in response rate (e.g., Leander, 1973; Powell \& Peck, 1969). The present effect is just the opposite. It represents a change in the rate of responding in the absence of a change in the rate of reinforcement.

Although the variables that produced the present functions are unknown, the present data can help to separate two broad classes of variables that may contribute. The present data suggest that factors related to reinforcement (e.g., satiation) exert more control over both the location of the peak of the function and the steepness of the descending limb of the function than do factors related to responding (e.g., fatigue).

Figure 5 presents critical data about the location of the peak of the function: keypressing for sweetened condensed milk peaked after approximately $20 \mathrm{~min}$, regardless of session duration. This was true even though the number of responses differed for different session lengths. There was a mean of 851.5 responses in the first eight components of the session ( $20 \mathrm{~min}$ ) for the 30 -min sessions, a mean of $1,292.9$ responses in the first four components (20 $\mathrm{min}$ ) for the 60-min sessions, and a mean of 1,061.0 responses in the first three components $(22.5 \mathrm{~min})$ for the 90-min sessions. Therefore, responding reached a peak after 20 reinforcers had been delivered, regardless of the number of responses. This, in turn, suggests that the ascending limb of the function is controlled more by reinforcer-related variables than by response-related variables.

A comparison of the results for the multiple VI 15-sec VI 15-sec and multiple VI 30-sec VI 30-sec schedules in Figure 1 provides critical data about the steepness of the descending limb of the function. The subjects responded faster on the multiple VI 30-sec VI 30-sec schedule (mean $=52.0$ responses per minute) than they did on the multiple VI 15-sec VI 15-sec schedule (mean $=35.1$ responses per minute), but they collected more reinforcers from the multiple VI 15-sec VI 15-sec schedule than from the multiple VI 30-sec VI 30-sec schedule. Figure 1 shows that the descending limb of the function was steeper for the multiple VI 15-sec VI 15-sec schedule. This suggests that higher rates of reinforcement contribute more to producing a steep function than do higher rates of responding.

The results presented in this paper raise more questions than they answer. The form of the present bitonic function should be quantified, and the factors that control this function should be determined. The generality of the bitonic function for different schedules, species, reinforcers, responses, and procedures should be determined. Finally, the implications of the present functions for theories of operant behavior should be investigated. Most theories use rate of responding averaged across the session as their dependent variable. Because the present results show that the average rate of responding may mask strong regularities in behavior at a more molecular level, the present results suggest that many theories of operant behavior are incomplete.

\section{REFERENCES}

AzRIN, N. H. (1960). Effects of punishment intensity during variableinterval reinforcement. Joumal of the Experimental Analysis of Behavior, 3, 123-142.

Azrin, N. H., Holz, W. C., * HAkE, D. F. (1963). Fixed-ratio punishment. Journal of the Experimental Analysis of Behavior, 6, 141-148.

Badia, P., Culeertson, S., \& Lewis, P. (1971). The relative aversiveness of signalled vs unsignalled avoidance. Journal of the Experimental Analysis of Behavior, 16, 113-121. 
BAUM, W. M. (1981). Optimization and the matching law as accounts of instrumental behavior. Joumal of the Experimental Analysis of Behavior, 36, 387-403.

Catania, A. C., \& Reynolds, G. S. (1968). A quantitative analysis of responding maintained by interval schedules. Journal of the Experimental Analysis of Behavior, 11, 327-383.

Dougan, J. D., \& MCSWEeney, F. K. (1985). Variation in Herrnstein's $r_{0}$ as a function of alternative reinforcement rate. Journal of the $E x$ perimental Analysis of Behavior, 43, 215-223.

FleshleR, M., \&offman, H. S. (1962). A progression for generating variable-interval schedules. Journal of the Experimental Analysis of Behavior, 5, 529-530.

ForeE, D. D., \& LoLorDo, V. M. (1970). Signalled and unsignalled free-operant avoidance in the pigeon. Joumal of the Experimental Anal. ysis of Behavior, 13, 283-290.

GRIFFIN, P., \& STEWART, D. J. (1977). Line-orientation generalization following signalled-reinforcer training. Journal of the Experimental Analysis of Behavior, 25, 151-160.

HAKE, D. F., \& AZRIN, N. H. (1965). Conditioned punishment. Journal of the Experimental Analysis of Behavior, 8, 279-293.

Hake, D. F., Azrin, N. H., OxFord, R. (1967). The effects of punishment intensity on squirrel monkeys. Joumal of the Experimental Analysis of Behavior, 10, 95-107.

HerRnstein, R. J. (1970). On the law of effect. Journal of the Experimental Analysis of Behavior, 13, 243-266.

Heyman, G. M. (1983). A parametric evaluation of the hedonic and motoric effects of drugs: pimozide and amphetamine. Journal of the Experimental Analysis of Behavior, 40, 113-122.

Hineline, P. N. (1978a). Warmup in avoidance as a function of time since prior training. Joumal of the Experimental Analysis of Behavior. 29, 87-103.

HiNeLiNe, P. N. (1978b). Warmup in free-operant avoidance as a function of the response-shock $=$ shock-shock interval. Joumal of the Experimental Analysis of Behavior, 30, 281-291.

Hodos, W., Leibowttz, R. W., \&onbright, J. C. JR. (1976). Nearfield visual acuity of pigeons: Effects of head location and stimulus luminance. Journal of the Experimental Analysis of Behavior, 25, 129-141.

Hoffman, H. S., Fleshler, M., \& Chorny, H. (1961). Discriminated bar-press avoidance. Journal of the Experimental Analysis of Behavior, 4, 309-316.

Hutchinson, R. R., Renfrew, J. W., \& Young, G. A. (1971). Effects of long-term shock and associated stimuli on aggressive and manual responses. Journal of the Experimental Analysis of Behavior, 15, 141-166.

LEANDER, J. D. (1973). Shock intensity and duration interactions on free-operant avoidance behavior. Journal of the Experimental Analysis of Behavior, 19, $481-490$.
Lyons, D. O., \& Ozouns, D. (1970). Pavlovian conditioning of shockelicited aggression: A discrimination procedure. Journal of the $E x-$ perimental Analysis of Behavior, 13, 325-331.

McSweeney, F. K., Dougan, J. D., Higa, J., \& Farmer, V. A (1986). Behavioral contrast as a function of component duration and baseline rate of reinforcement. Animal Learning \& Behavior, 14, 173-183.

McSweeney, F. K., Hatfield, J., \& Allen, T. M. (1990). Withinsession responding as a function of post-session feedings. Behavioural Processes, 22, 177-186.

McSweeney, F. K., Hinson, J. M. (in press). Within-session patterns of responding. Joumal of the Experimental Analysis of Behavior.

MCSWeeney, F. K., Melville, C. L. (1990). Behavioral contrast as a function of component duration for lever pressing using a withinsession procedure. Animal Learning \& Behavior, 19, 71-80.

Muscio, B. (1921). Is a fatigue test possible? British Journal of Psychology, 12, 31-46.

OLDS, J. (1956). Runway and maze behavior controlled by basomedial forebrain stimulation in the rat. Joumal of Comparative \& Physiological Psychology, 49, 507-512.

OldS, J., MiLner, P. (1954). Positive reinforcement produced by electrical stimulation of septal area and other regions of the rat brain. Joumal of Comparative \& Physiological Psychology, 47, 419-427.

PoWELL, R. W. (1970). The effect of shock intensity upon responding under a multiple-avoidance schedule. Joumal of the Experimental Analysis of Behavior, 14, 321-329.

Powell, R. W., PECK, S. (1969). Running-wheel activity and avoidance in the Mongolian gerbil. Joumal of the Experimental Analysis of Behavior, 12, 779.787.

RuBiN, H. B., Brown, H. J. (1969). The rabbit as a subject in behavioral research. Journal of the Experimental Analysis of Behavior, 12, 663-667.

Spealman, R. D., Gollub, L. R. (1974). Behavioral interactions in multiple variable-interval schedules. Journal of the Experimental Analysis of Behavior, 22, 471-481.

Staddon, J. E. R. (1967). Attention and temporal discrimination: Factors controlling responding under a cyclic-interval schedule. Journal of the Experimental Analysis of Behavior, 10, 349-359.

STADDON, J. E. R. (1979). Operant behavior as adaptation to constraint. Journal of Experimental Psychology: General, 108, 48-67.

WERTHEIM, G. A. (1965). Some sequential aspects of IRTs emitted during Sidman-avoidance behavior in the white rat. Joumal of the Experimental Analysis of Behavior, 8, 9-15.

(Manuscript received June 5, 1991; revision accepted for publication October 30, 1991.) 